\title{
Stress reduction intervention and the initial periodontal therapy outcome in chronic periodontitis patients. a randomized controlled pilot study
}

\author{
Karthikeyan Bangalore Varadhan ${ }^{1 *}$, Munaga Shanmukha Venkata Nagarjuna ${ }^{1}$, Prabhuji Munivenkatappa \\ Lakshmaiah Venkatesh ${ }^{1}$, Sai Jyothsna ${ }^{1}{ }^{1}$, Smiti Bhardwaj ${ }^{1}$, Rekha Murahari ${ }^{2}$ \\ ${ }^{1}$ Dept. of Periodontology, Krishnadevaraya College of Dental Sciences and Hospital, \\ Rajivgandhi University of Health Sciences, Bangalore, India \\ ${ }^{2}$ MBBS, DPM (Diploma in Psychological Medicine), District Hospital. Sangareddy, Telangana \\ *Corresponding author E-mail: drkarthikeyanbv@gmail.com
}

\begin{abstract}
Background: Psychosocial stress, have been implicated as risk indicators for periodontal disease. Minimal evidence in literature exists to assess the effect of stress reduction therapy (SRT) in the outcome of non-surgical periodontal therapy (NSPT).

Objective: Hence, we aimed to explore the possibility of employing SRT in stressed patients as an adjunctive intervention in the management NSPT.

Materials and Methods: Sixty male patients divided into Group 1 comprised of 20 unstressed chronic periodontitis patients receiving nonsurgical periodontal therapy alone, Group $2 \mathrm{a}$ (20 stressed chronic periodontitis patients) received intervention focused on SPT with NSPT and Group $2 b$ (20 stressed chronic periodontitis patients) received only NSPT without SPT. Their stress level was evaluated using a standard questionnaire method and salivary cortisol levels, at baseline and 3 months along with periodontal parameters.

Results: There was significant reduction in stress parameters for Group 2a patients. The reduction of total periodontitis affected sites was highest in Group I patients (55.4\%), followed by Group 2a patients (53.2\%) and Group 2b patients (38\%).

Conclusion: SRT may result in comparable treatment outcome to unstressed chronic periodontitis patients. This study opens new avenues to investigate the effects of stress management as an adjunctive to conventional NSPT, which should be explored further.
\end{abstract}

Keywords: Derogatis Stress Profile Scores; Non-Surgical Periodontal Therapy; Periodontal Disease; Psychological Stress; Stress Reduction Therapy; Salivary Cortisol.

\section{Introduction}

Periodontitis is a multifactorial disease where microbial dental biofilms are considered to be a key etiological agent for the initiation \& progression of the inflammatory process (Genco, 1996; Page and Beck, 1997; Page. 1998; Elter et al., 1999). Apart from the microbial biofilms, several other risk factors have been associated with increased susceptibility, progression and severity of periodontal diseases such as systemic diseases, genetic polymorphisms, socio-economic or educational status, tobacco smoking, oral hygiene level and psychological stress (Reners and Breex, 2007; Breivik et al., 1996).

With respect to other possible factors influencing chronic periodontitis, more direct evidence has emerged that stress, depression and anxiety contribute to the development of periodontitis in odds ratio of 1:2. Further, it has been shown that patients with stress are more prone to develop periodontal disease than patients without stress (Seiffert et al., 2002; Breivik, 2001; Bartold and Kylstra, 1994; KiecoltGlaser et al., 2003). It is speculated that chronic stress contribute to the development of periodontitis by having a net negative effect on the immunological response of body or by health related risk behaviors such as smoking, over eating and lessen compliance with the preventive behavior or even both (Leresche and Dworkin, 2002). In addition, it has been found that patients experiencing stress were slower in recovery from periodontal treatment compared to patients who are not experiencing stress (Leresche and Dworkin, 2002).

Interestingly, the impact of stress on the pathogenesis and periodontal treatment outcomes depends upon the individuals coping ability. Literature evidence shows that emotional-focused coping individuals (defensive coping, resigned coping, distractive coping which are advantageous in the short term) have more advanced disease and poor response to non-surgical periodontal treatment when compared to problem focused coping (i.e. active coping) (Wimmer et al., 2002). Hence, assessment of a patient's stress level, their coping ability and stress management might be of value in understanding psychological effects on periodontal health and its disease process, which will be helpful in future preventive care. Considering these facts, we hypothesize that if stress is causally related to the worsening of parameters in a chronic periodontitis patient, its alleviation might result in an additive response to the conventional periodontal therapy. However, till date, there are no intervention studies on possibility of employing psychological intervention (stress reduction therapy (SRT)) as adjunctive measure in the treatment of periodontitis patients with unfavorable psychological background. Hence, considering this hypothesis, the present study is conducted which is first of its kind, to explore and evaluate, if intervention focused on stress management enhancement

Copyright $\odot$ Karthikeyan Bangalore Varadhan et al. This is an open access article distributed under the Creative Commons Attribution License, which permits unrestricted use, distribution, and reproduction in any medium, provided the original work is properly cited. 
training may serve as adjunctive role in non-surgical treatment of periodontal diseases by monitoring the improvements in periodontal condition through clinical parameters and correlating with stress marker like salivary cortisol levels and Derogatis stress profile (DSP) scores.

\section{Materials and methods}

\subsection{Source of data}

It is a randomized control parallel study. The study population consisted of randomly selected sixty, systemically healthy, male patients selected out of 150 screened patients (with exclusion of 90 patients) who were screened at the Border Security Force, Yelahanka, Bangalore. The study was conducted during the period from March 2015 to November 2015. They were recruited for the study when they satisfied the following inclusion and exclusion criteria.

Inclusion criteria includes, patients within the age group of 30 to 55 years, systemically healthy individuals with chronic periodontitis and who were co-operative and willing to attend follow up visits

Exclusion criteria includes, patients on corticosteroids and antipsychotic drug therapy, who had received any periodontal therapy, surgical or non- surgical within the past six months of baseline examination, Smokers, no prior history of non-surgical periodontal therapy within 6 months, with less than 20 permanent teeth remaining, history of psychiatric treatment within past 6 months, known systemic diseases and conditions.

All eligible patients, who volunteered, were informed of the nature, potential risks and benefits of their participation in the study and a written signed informed consent was obtained from whom who agreed to participate. The ethical clearance for the study was obtained from the institutional ethical committee of Krishnadevaraya College of Dental Sciences and Hospital, affiliated to the Rajiv Gandhi University of Health Sciences, Bangalore, India.

\subsection{Criteria for subject grouping}

Based on the clinical parameters like modified gingival index (GI; Loe and Silness, 1963), Plaque Index (PI; Silness and Loe, 1964), periodontal probing depth (PPD), clinical attachment level (CAL), bleeding on probing (BOP; Ainamo and Bay, 1975), salivary cortisol levels and Derogatis stress profile (DSP; Derogatis, 1995), sixty subject populations were categorized into two groups. The study population of chronic generalized periodontitis subjects were selected according to the criteria proposed by the 1999 International World Workshop for a Classification of Periodontal Diseases and Conditions: chronic generalized periodontitis subjects had moderate to severe alveolar bone loss ( $\mathrm{CAL} \geq 3 \mathrm{~mm}$ and $\mathrm{PPD} \geq 5 \mathrm{~mm})$ in multiple sites of all four quadrants of the mouth but with no evidence of rapid progression.

Group 1: Comprises of 20 chronic generalized periodontitis unstressed patients receiving non-surgical periodontal therapy (scaling and root planing) alone.

All patients who showed clinical signs of gingival inflammation and attachment loss, gingival index $\geq 2$, plaque index $2.0-3.0$, gingival bleeding index : > 1, bleeding present on probing within 10 seconds.

Group 2: Comprises of 40 chronic periodontitis patients associated with stress and clinical signs of gingival inflammation and attachment loss (gingival index $\geq 2$, plaque index $2.0-3.0$, gingival bleeding index: $>1$, bleeding on probing within 10 seconds, $P P D \geq 5 \mathrm{~mm}$, clinical attachment level $\geq 3 \mathrm{~mm}$, Radiographic evidence of bone loss, Derogatis stress profile 77 questionnaire score 0.72 - 0.90 , cortisol levels > $2.5 \mathrm{ng} / \mathrm{ml}$ (AM; stressed).

They were further subdivided by simple randomization of computer software program that generates the random sequence method into two sub groups of 20 each, based on the intervention (SRT): enclose assignments in sequentially numbered, sealed envelopes. Examiner (1) generated random allocation sequence and carried out post intervention clinical examination of periodontal parameters, second examiner (2) Enrolled the participants for the study when they satisfied the inclusion and exclusion criteria. Carried out case history taking, initial clinical examination of periodontal parameters and radiographic examination to group the participants, third examiner (3) assigned participant to intervention and examiner (4) performed scaling and root planning, examiner (5) performed supportive periodontal therapy and examiner (6) performed and evaluated the stress reduction therapy. It is a double blind study where all the examiners and participants, both were blinded.2a: Comprises of 20 stressed chronic periodontitis patients associated with chronic stress, who received intervention focused on SRT along with non-surgical periodontal therapy. 2b: Comprises of 20 stressed chronic periodontitis patients associated with chronic stress who received only non-surgical periodontal therapy (SRP) without intervention focused on SRT.

A sequential flow chart for the study protocol is shown in Figure.1. 


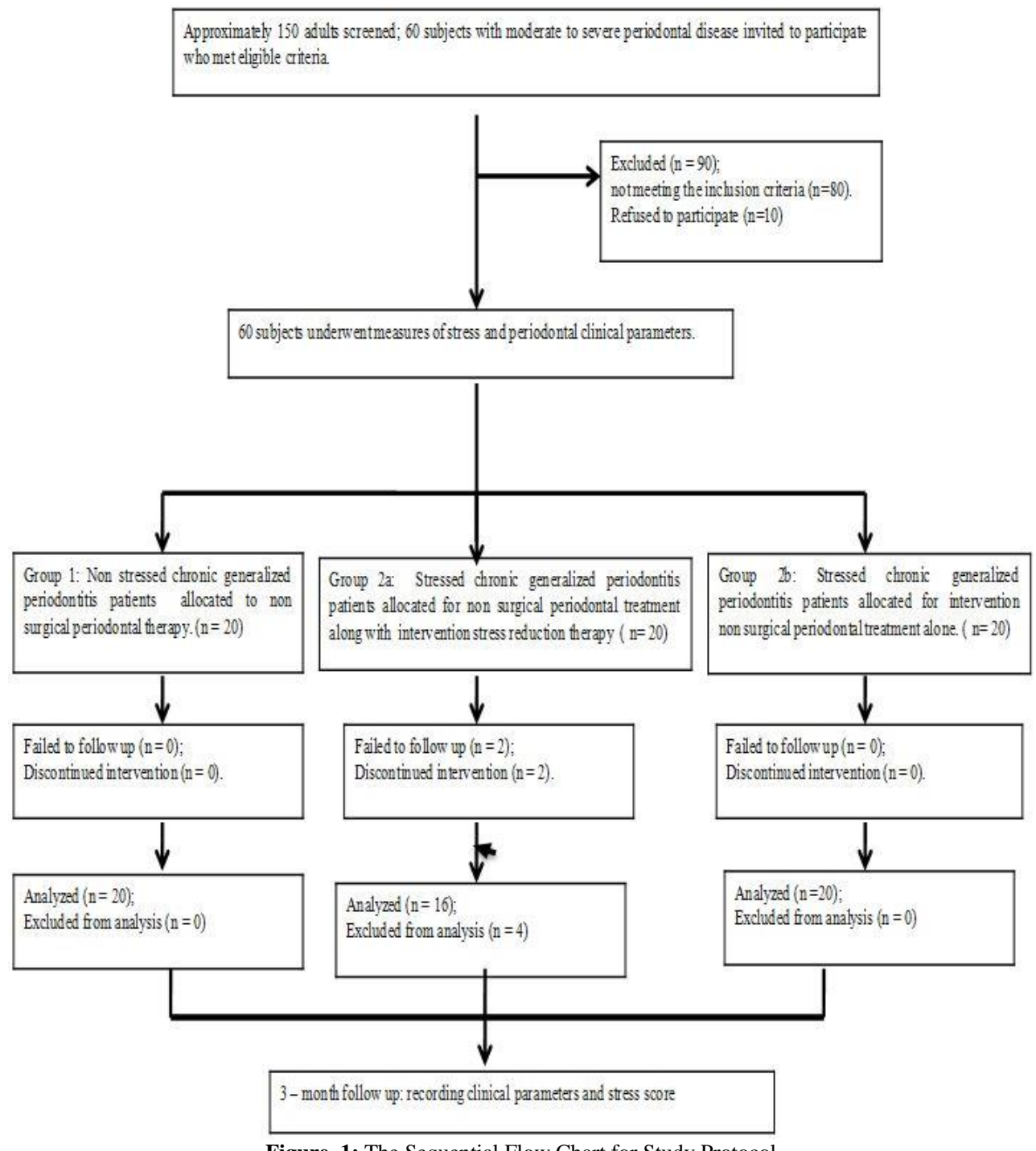

\subsection{Pre-sampling clinical evaluation of patients}

Patients were selected for each group after a brief and precise case history recording that included patient's chief complaint, clinical examination and radiographic evaluation. Demographic characteristics, such as age, sex, diet and medical history were also recorded.

\subsection{Systemic examination}

Assessment of Blood pressure (BP), Body Mass Index (BMI) and hematological testing for Fasting Blood Sugar (FBS) was carried out for all the patients, to rule out the presence of unidentified systemic conditions such as cardiovascular diseases and diabetes mellitus, which can potentially influence cortisol levels. The BP was recorded by using a mercury sphygmomanometer. BMI was evaluated by use of standard height and weight scales (Misra et al., 2009).

\subsection{Intra-oral examination}

Clinical assessment of the oral hygiene and gingival status was done by plaque index, bleeding on probing index and gingival index. These indices were repeated at 90th day for assessment of oral hygiene maintenance and presence or absence of gingival inflammation. For each subject full-mouth periodontal probing and charting was recorded using UNC-15 probe. PPD and CAL were measured on six sites (mesiobuccal, mid-buccal, disto-buccal, mesio-lingual, mid-lingual and disto-lingual) per tooth using UNC-15.

The measurement of REC (recession), PPD and clinical attachment level (CAL) was done according to Pilgram et al., (2000). REC was measured from the CEJ to the gingival margin, with a positive value if there was recession and a negative value in the absence of recession; CAL was calculated by summation of PPD and REC. The clinical diagnosis of chronic periodontitis was made based on the criteria described by Armitage, 1999. CAL was stratified into 3 ordered categories: slight: $1-2 \mathrm{~mm}$, moderate: 2 - 4 , severe: $>5 \mathrm{~mm}$.

\subsection{Examiner calibration for periodontal examination}

Before recording probing depths calibration exercise for clinical parameters was performed in five patients before the actual study. Examiners 1 and 2 conducted the recordings for clinical parameters at 1 week interval. The order of patients was changed in between the examinations, by a third examiner (3) who was blinded to the whole process. The periodontal probing depth estimation was judged to be reproducible if the intra and inter-examiner agreement within $\pm 1 \mathrm{~mm}$ between repeated measurements was at least $80 \%$. The kappa value for 
intra-examiner agreement for examiner 1 and 2, between the two measurements was recorded to be 0.91 and 0.94 respectively. The interexaminer calibration was recorded to be 0.84 .

The above-mentioned parameters were recorded at baseline and at follow-up visits scheduled at 90th day, following initial therapy by prealigned and assessed examiners BVK.

\subsection{Stress assessment}

The Derogatis stress profile (DSP) is a self-report measure designed to assess chronic stress. This 77 -item inventory assesses 11 components of stress, including vocational environment, domestic environment, health environment, time pressure, driven behavior, attitude posture, relaxation potential, role definition, hostility, anxiety, and depression. Participants rated each statement from 0 (not at all true of me) to 4 (extremely true of me). The DSP contains items such as, I take some time out almost every day just to relax, and I am usually worried about something.

Stressed and non-stressed patients were differentiated by Derogatis stress profile 77 questionnaire. The scale takes approximately $12-13$ minutes to complete. Stress score range is $0.72-0.90$. $(<0.72-$ unstressed, 0.72 to $0.90-$ stressed, $0.90-$ severe stressed $)$

A professional recorded the clinical data before commencing stress reduction therapy. The stress questionnaires were issued by psychologist involved in psychological assessment \& analysis and who is fluent in local language and English. Instructions were explained and patients were arranged to complete the questionnaires in a calm environment. The examiner 1 was standby to clarify any queries arising during completion of the questionnaires. All the questionnaires were designed in self-administered format. For those illiterate or marginally literate patients, who were mainly from the older age groups, their questionnaires were completed in an interviewer assisted format.

Patients were asked to complete a questionnaire including the following sections: 1) demographic and socio-economic details; 2) medical history - reporting symptom and diagnosed systemic diseases; 3) dental habits and dental care utilization; and 4) history of cigarette smoking and exposure to occupational hazards. Patients were then given a set of self-administered stress questionnaires in a face-to-face interview with examiner 1 .

Salivary sample collection was done according to the passive drool method (Dawes et al., 2000) and samples were collected in plastic vials and centrifuged for $20 \mathrm{~min}$ at $3000 \mathrm{rpm}$ to remove bacteria and cellular debris. The supernatant was pipetted out and stored at $-20^{\circ} \mathrm{C}$ for further analysis.

\subsection{Intervention therapy}

\subsubsection{Periodontal treatment}

All patients of Group 1 and Groups $2 \mathrm{a}$ and $2 \mathrm{~b}$ were first submitted to the hygiene phase of the periodontal therapy, which includes supragingival plaque and calculus removal, provisional restoration, and the removal of overhanging fillings. Scaling and root planing (SRP) was performed under local anesthesia (2\% lignocaine hydrochloride with 1:200000 adrenaline) using area specific Gracey periodontal curettes and an ultrasonic device. Within the duration of the study, all patients received supportive therapy, which included professional plaque control and oral hygiene information.

The treatment was concluded within 7 days without the use of antibiotics or local antimicrobials. The patients were monitored by assessment of clinical parameters at baseline and at 90th day of therapy.

\subsubsection{Stress reduction therapy}

After the assessment of clinical, stress and coping parameters and completion of non- surgical periodontal therapy, intervention for stress reduction was commenced for Group 2a patients through transactional model protocols.

Transactional model is an adept framework for treatment and evaluation of stress through problem solving and emotional coping methods. It is considered as a treatment of choice for stressed patients confronting both unmanageable and manageable stressor events. The patient' $\mathrm{s}$ evaluation of potential gravity of the situation (primary appraisal) and his/her ability to beneficially alter the situations and emotions (secondary appraisal), both play a crucial part in assessing the coping efforts which are required and their obtained outcomes. Since, the use of Transactional model adjudicates the degree, duration and type of coping efforts required, it was used to assess, evaluate and accordingly counsel the stressed patients.

As a part of the Transactional Model modus operandi, a course of seven sessions were held for Group 2a patients, distributed uniformly over a period of six months (two sessions per month, totally 7 sessions). These sessions of approximately 45 minutes duration, were held in a setting of calm and pleasant environment.

Session 1: Day 0

The subjects ( 5 patients at a time) were educated for better impact of our intervention regarding stress, its primary etiologic agent and its ill effects on periodontal \& systemic health through lectures, images and video tapes (Powerpoint slides). Further, patients were motivated to employ stress reduction protocols such as assertion training via diary writing and relaxation techniques including breathing exercises, meditation, yoga, musical detours etc., as a daily practice. To keep a check on patient compliance, the patients were provided with a pamphlet, pictorially depicting the protocols they were supposed to follow. They were supposed to mark a tick against the stress reduction practices they followed each day. No compulsion was made for them to strictly adhere to these practices, to avoid additional stress. The patients were asked to bring back the marked pamphlet in the next session.

Session 2: Day 15

In the next session (15th day), the patient's ability to manage and alter the stressful situation was assessed through cope questionnaire individually along with explanation of the benefits of adequate coping with stress (Stress reduction techniques reinforcement). The patient compliance to the suggested protocol was evaluated by assessing their daily attempts towards stress reduction, through the pamphlet they were asked to bring back. Execution of even one stress reduction protocol daily was considered as a positive marker for subjective compliance and improvisation.

Subjects unwilling to follow the stress reduction protocols were excluded from the study. Motivational reinforcement of the coping measures was carried out for subjects, who were unable to adhere to the protocol due to personal problems. Telephonic communications with patients were also made to remotely observe and guide the subjects through the protocol.

Session 3: Day 30 
This session was held separately for each patient. Herein, in addition to the reinforcement of coping behavior, the patients were advised to actively cope with the stressor by modulating their attitude towards the stressful situation. The coping method was selected for the patients based on their stressor situations.

In manageable stressor incidences, coping strategies directed at changing a stressful situation were advised to the patient (problem solving coping). As an example, the subject A 36 years old man, had faced a problem of financial crisis to pay the hire for his vehicle and home because of his inability or severe illness and because of that situation he is worried and tensed about that situation which caused stress mentally to guide him towards smoking, drinking and other habits which are injurious to systemic health. Because of this financial stress, ignore concern towards his family, health and surrounding environment and he considered himself as he cannot solve his problem. In cases of unmanageable stressor incidences, coping strategies directed towards an alternative solutions to their problems, such as obtaining advices from close associates, confiding in trustworthy acquaintances and availing assistance from supporters were suggested (emotional coping).Ex: As an example, the subject A 32 year's old man had faced a problem of sudden death of his father and mother in an accident. He felt that himself as an isolated from family and loving ones and went into depression along with ignoring health and addicted to bad habits such as drinking, smoking to forget that worst situation because of his inability to manage his rest of life without them.

Sessions 4, 5 \& 6: Day 45, 60 and 75

Reinforcement of the benefits of adequate coping with stress (Stress reduction techniques reinforcement). In addition, Oral Hygiene instructions were concreted to the patients.

Session 7: Day 90

In this session, Group 1, 2a and $2 \mathrm{~b}$ patients were recalled back. The Group $2 \mathrm{a}$ and $2 \mathrm{~b}$ patient's ability to manage and alter the stressful situation was assessed through cope questionnaire. The compliance of Group 2a subjects to stress reduction practices was judged through pamphlets, which they were supposed to bring back.

The reassessment for the stressed subjects was carried out after every 15 days by the examiner 1 within $13-15$ minutes of time period and interpreting the values by the patient's selected answers in the followed scale and analyzing whether the patients are following or not about our therapy protocol and what actually there are doing. This included their evaluation for use of coping techniques and reinforcement of stress reduction protocols.

\section{Biochemical assessment of cortisol}

Samples were assayed for cortisol levels by using chemiluminescence immunoassay (CLIA) by microplateluminometers provides a sensitive, high throughput and economical alternative to conventional colorimetric methodologies, such as enzyme-linked immunosorbent assays (ELISA).. This method has a reported sensitivity of $<0.5 \mu \mathrm{g} / \mathrm{ml}$. Results were reported as the total amount in micrograms per milliliter of sample $(\mu \mathrm{g} / \mathrm{ml})$.

\subsection{Method of statistical analysis}

One-way analyses of variance were used to test the difference between groups. Analysis of Variance is a technique by which the total variation is split into two parts one between groups and the other within the groups. The Student ' $t$ ' test was used to determine whether there was a statistical difference between groups in the parameters measured. The association between clinical parameter and DSP and salivary cortisol were assessed by PERSONS CORRELATION coefficient. Normality of data was tested using Shapiro-Wilk test. The results were averaged (mean + standard deviation) for continuous data are presented in Table 1 to 4 . In the entire above test $p$ value less than 0.05 were taken to be statistically significant. The data was analyzed using SPSS package (ver 10.5).

\section{Results}

From the 150 subjects enrolled in this randomized control parallel study from March 2015 to November 2015, 150 were screened and 60 were included in the present study. Approximately $54 \%(\mathrm{~N}=80)$ of the enrolled subjects were lost to not meeting the inclusion criteria, $6.7 \%(\mathrm{~N}=10)$ were refused to participate, in included subjects $3.3 \%(\mathrm{~N}=2)$ were lost to follow up and $3.3 \%(\mathrm{~N}=2)$ were discontinued intervention (Fig. 1). Power of the study is $80 \%$ and the estimated effect size and precision was set at $95 \%$ confidence interval.

Included 40 patients who were randomly assigned into Group 2a (20) and Group $2 \mathrm{~b}(20)$. All the participants received intended intervention (SRP) and where analyzed for the primary outcome (improvement in Clinical Parameters). When compared from the baseline, a significant reduction was noted at three months in the mean GI and PI scores for group 2 a once only $(\mathrm{p}<0.001)$. However, there was a significant reduction in BOP in all the groups from baseline to 3 months $(\mathrm{p}<0.001)$.

Analytical assessment for the mean number of PD sites for all the groups revealed a significant reduction after 3 months of intervention ( $\mathrm{p}$ $<0.001)$. At end of 3 months, the highest overall reduction in pocket depth was seen in Group $1(2.32 ; \mathrm{p}<0.001)$, least in Group 2b $(0.29$; $\mathrm{p}>0.05)$ and intermediate in Group $2 \mathrm{a}(1.95 ; \mathrm{p}<0.001)$. Likewise improvement in the CAL levels was significantly higher in Group 1 $(1.16 ; p<0.001)$ and least in Group $2 \mathrm{~b}(0.15 ; \mathrm{p}>0.05)$ but Group 2a values $(1.11 ; \mathrm{p}<0.001)$ were intermediate between the highest and lowest values. These values suggest that after periodontal and psychological intervention there is a significant improvement in the clinical parameters and stress levels of Group 2a chronic stressed periodontitis patients when compared with the Group 2b chronic stressed periodontitis patients without any stress reduction procedure.

When the mean gain in the CAL was assessed among Groups 1, 2a and 2b, using ANOVA test, the difference of mean was shown to be statistically significant at baseline with $\mathrm{p}$ value $<0.001$. Further pairwise intergroup analysis by t-test revealed that at the end of 3 months, significant gain in the sites with CAL was obtained in all the groups except on $\geq 5 \mathrm{~mm}$ group of Group $2 \mathrm{~b}$.

A weak positive correlation between DSP scores and CAL found in the Group 2a of $3-4 \mathrm{~mm}$, Group 1 and Group $2 \mathrm{a}$ of $\geq 5 \mathrm{~mm}$ the at the base line and end of the third month and between the salivary cortisol levels and pocket depths at the base line and end of the third month, a weak positive correlation found in the Group $2 b$ of $4-6 \mathrm{~mm}$, Group 1 and Group $2 \mathrm{~b}$ of $>8 \mathrm{~mm}$. But a strong positive correlation found in the Group 2a of $4-6 \mathrm{~mm}$ and $>8 \mathrm{~mm}$. And also weak positive correlation between the salivary cortisol levels and CAL found in the Group 1 of $1-2 \mathrm{~mm}$, Group $2 \mathrm{~b}$ of $\geq 5 \mathrm{~mm}$. But a strong positive correlation found in the Group $2 \mathrm{a}$ of $3-4 \mathrm{~mm}$ and Group $1 \mathrm{of} \geq 5 \mathrm{~mm}$ at the base line and end of the third month.

In summary, there was a significant mean reduction in salivary cortisol levels, DSP scores, GI, PI, BOP, PD, CAL of Group 2a when stress reduction therapy with periodontal intervention were given when compared to other groups, where only periodontal intervention was given. This indicates in patients under psychological stress when SRT was used as an adjunctive, there was a statistically significant outcome to NSPT (Table 1-4 \& Figures 2,3). There was no associated untoward effects in all the study groups.

Table 1: The Mean Values, Standard Deviation (SD), Minimum and Maximum Values for Salivary Cortisol Levels and DSP Scores 


\begin{tabular}{|c|c|c|c|c|c|c|c|c|c|c|}
\hline \multirow[t]{2}{*}{ Salivary Cortisol Levels } & \multicolumn{5}{|c|}{ Baseline } & \multicolumn{5}{|c|}{3 months } \\
\hline & $\mathrm{N}$ & Mean & SD & Min. & Max. & $\mathrm{N}$ & Mean & SD & Min. & Max. \\
\hline Group 1 & 20 & 2.12 & 0.25 & 1.65 & 2.46 & 20 & 1.98 & 0.20 & 1.51 & 2.31 \\
\hline Group 2a & 20 & 3.46 & 0.69 & 2.52 & 5.10 & 16 & 2.60 & 0.58 & 1.78 & 2.99 \\
\hline Group 2b & 20 & 3.26 & 0.50 & 2.55 & 4.50 & 20 & 2.85 & 0.31 & 2.35 & 3.54 \\
\hline \multirow[t]{2}{*}{ DSP Scores } & \multicolumn{5}{|c|}{ Baseline } & \multicolumn{5}{|c|}{3 months } \\
\hline & $\mathrm{N}$ & Mean & SD & Min. & Max. & $\mathrm{N}$ & Mean & SD & Min. & Max. \\
\hline Group 1 & 20 & 0.48 & 0.13 & 0.25 & 0.71 & 20 & 0.46 & 0.12 & 0.28 & 0.70 \\
\hline Group 2a & 20 & 2.25 & 0.56 & 1.10 & 2.94 & 16 & 1.35 & 0.39 & 0.98 & 2.11 \\
\hline Group $2 b$ & 20 & 1.95 & 0.60 & 0.90 & 2.73 & 20 & 1.84 & 0.57 & 0.91 & 2.72 \\
\hline
\end{tabular}

Table 2: The Mean Values, Standard Deviation (SD), Minimum and Maximum Values for PD, CAL

\begin{tabular}{|c|c|c|c|c|c|c|c|c|}
\hline & Group & & Mean & SD & Min & Max & "P" value & Mean diff \\
\hline \multirow{6}{*}{ PD } & \multirow{2}{*}{ Group 1} & Baseline & 6.307 & 1.9172 & .0 & 9.0 & \multirow{2}{*}{$<0.001 *$} & \multirow{2}{*}{2.32} \\
\hline & & 3 month & 3.980 & 1.4872 & .0 & 7.5 & & \\
\hline & \multirow{2}{*}{ Group $2 \mathrm{a}$} & Baseline & 5.377 & 1.6352 & 3.2 & 8.4 & \multirow{2}{*}{$<0.001 *$} & \multirow{2}{*}{1.95} \\
\hline & & 3 month & 3.428 & 1.2045 & 1.5 & 5.9 & & \\
\hline & \multirow{2}{*}{ Group $2 b$} & Baseline & 6.982 & 1.3384 & 4.7 & 9.2 & \multirow{2}{*}{$<0.001 *$} & \multirow{2}{*}{0.29} \\
\hline & & 3 month & 6.698 & 1.5379 & 3.4 & 9.1 & & \\
\hline \multirow{6}{*}{ CAL } & \multirow{2}{*}{ Group 1} & Baseline & 3.174 & 1.7350 & .0 & 6.6 & \multirow{2}{*}{$<0.001$} & \multirow{2}{*}{1.16} \\
\hline & & 3 month & 2.013 & 1.4022 & .0 & 5.0 & & \\
\hline & \multirow{2}{*}{ Group 2a } & Baseline & 3.672 & 1.6692 & 1.4 & 6.6 & \multirow{2}{*}{$<0.001 *$} & \multirow{2}{*}{1.11} \\
\hline & & 3 month & 2.468 & 1.4199 & .3 & 4.3 & & \\
\hline & \multirow{2}{*}{ Group $2 b$} & Baseline & 3.763 & 1.7460 & 1.4 & 6.7 & \multirow{2}{*}{$<0.001 *$} & \multirow{2}{*}{0.15} \\
\hline & & 3 month & 3.610 & 1.7886 & 1.2 & 6.6 & & \\
\hline
\end{tabular}

- Statistically significant.

Table 3: The Mean Values, Standard Deviation (SD), Minimum and Maximum Values for GI, BOP, PI

\begin{tabular}{|c|c|c|c|c|c|c|c|c|c|c|}
\hline \multirow[t]{2}{*}{ GI } & \multicolumn{5}{|l|}{ Baseline } & \multicolumn{5}{|c|}{3 months } \\
\hline & Mean & $\mathrm{SD}$ & Median & Min. & Max. & Mean & SD & Median & Min. & Max. \\
\hline Group 1 & 1.224 & 0.355 & 1.115 & 0.77 & 1.91 & 1.042 & 0.355 & 1.000 & 0.53 & 1.81 \\
\hline Group 2a & 1.476 & 0.366 & 1.420 & 0.87 & 2.07 & 1.055 & 0.320 & 1.010 & 0.32 & 1.61 \\
\hline Group $2 b$ & 1.116 & 0.160 & 1.075 & 0.97 & 1.66 & 1.045 & 0.127 & 1.010 & 0.87 & 1.46 \\
\hline \multirow[t]{2}{*}{ BOP } & Baseline & & & & & $3 \mathrm{mont}$ & & & & \\
\hline & Mean & $\mathrm{SD}$ & Median & Min. & Max. & Mean & $\mathrm{SD}$ & Median & Min. & Max. \\
\hline Group 1 & 4.433 & 1.045 & 4.895 & 2.10 & 5.70 & 1.695 & 0.768 & 1.610 & 0.69 & 3.33 \\
\hline Group 2a & 4.567 & 0.996 & 4.985 & 2.12 & 5.71 & 1.755 & 0.646 & 1.765 & 0.80 & 2.76 \\
\hline Group $2 b$ & 4.531 & 0.933 & 4.895 & 2.12 & 5.71 & 3.414 & 1.046 & 3.670 & 1.28 & 5.09 \\
\hline \multirow[t]{2}{*}{ PI } & Baseline & & & & & $3 \mathrm{mont}$ & & & & \\
\hline & Mean & SD & Median & Min. & Max. & Mean & $\mathrm{SD}$ & Median & Min. & Max. \\
\hline Group 1 & 1.559 & 0.688 & 1.370 & 0.33 & 2.75 & 1.330 & 0.609 & 1.175 & 0.32 & 2.20 \\
\hline Group 2a & 1.747 & 0.546 & 1.720 & 0.91 & 2.89 & 1.211 & 0.374 & 1.025 & 0.71 & 1.89 \\
\hline Group $2 b$ & 1.652 & 0.404 & 1.620 & 1.04 & 2.29 & 1.603 & 0.394 & 1.585 & 1.01 & 2.20 \\
\hline
\end{tabular}

Table 4: Results of Pearson'S Correlation Coefficient (R) Test Compare Salivary Cortisol Level, Pocket Depths and Clinical Attachment Levels within the Groups at Baseline and End of Third Month

\begin{tabular}{|c|c|c|c|c|}
\hline \multicolumn{5}{|c|}{ Pearson's correlation coefficient (r) test compare salivary cortisol level and pocket depths } \\
\hline \multirow{3}{*}{ Baseline } & & Group 1 & Group $2 \mathrm{a}$ & Group $2 b$ \\
\hline & $\mathrm{R}$ & -0.122 & 0.093 & -0.267 \\
\hline & p'value & 0.608 & 0.697 & 0.256 \\
\hline \multirow[t]{2}{*}{3 month } & $\mathrm{R}$ & -0.139 & -0.445 & -0.141 \\
\hline & p'value & 0.560 & 0.084 & 0.553 \\
\hline \multicolumn{5}{|c|}{ Pearson's correlation coefficient (r) test compare salivary cortisol level and clinical attachment levels } \\
\hline \multirow[t]{2}{*}{ Baseline } & $\mathrm{R}$ & -0.047 & 0.166 & 0.280 \\
\hline & p'value & 0.843 & 0.484 & 0.232 \\
\hline \multirow[t]{2}{*}{3 month } & $\mathrm{R}$ & -0.214 & 0.498 & -0.018 \\
\hline & p'value & 0.366 & 0.049 & 0.940 \\
\hline
\end{tabular}

Pearson coefficient of correlation.

If the (r) value.

Is -1 , there is a perfect negative correlation.

Falls between -1 and -0.5 , there is a strong negative correlation.

Falls between -0.5 and 0 , there is a weak negative correlation.

Is 0 , there is no correlation.

Falls between 0 and 0.5 , there is a weak positive correlation.

Falls between 0.5 and 1 , there is a strong positive correlation.

Is 1 , there is a perfect positive correlation between the 2 sets of data. 

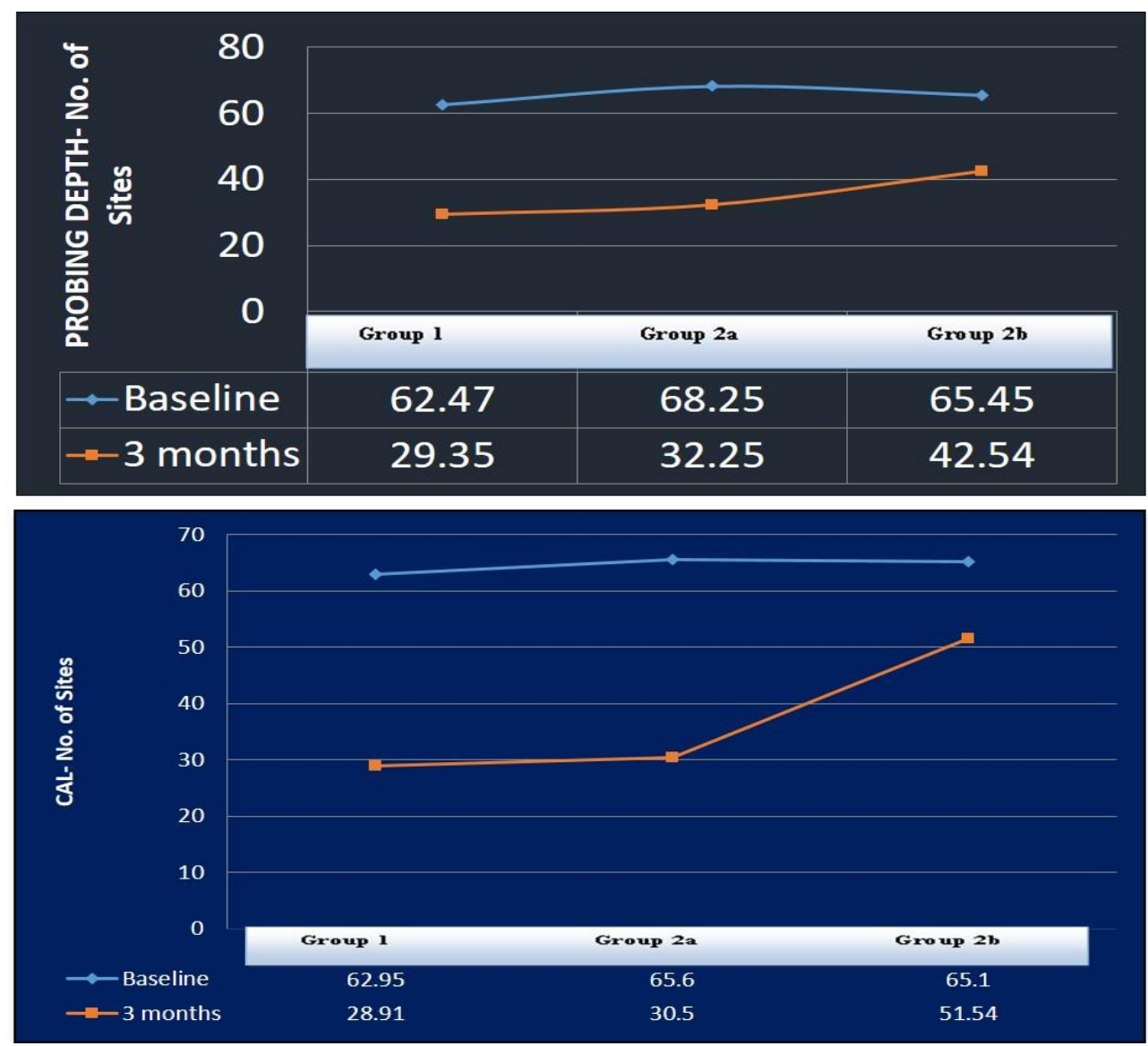

Fig. 2: Correlation of Pocket Depths and Clinical Attachment Levels (No. of Sites) in All the Groups from Baseline to 3 Months.

Group 1: Non stressed Chronic generalized periodontitis subjects with only NSPT intervention (NSCGP-NSPT).

Group 2a: Stressed Chronic generalized periodontitis subjects with NSPT and SRT intervention (SCGP-NSPT \& SRT).

Group 2b: Stressed Chronic generalized periodontitis subjects with only NSPT intervention (SCGP-NSPT).

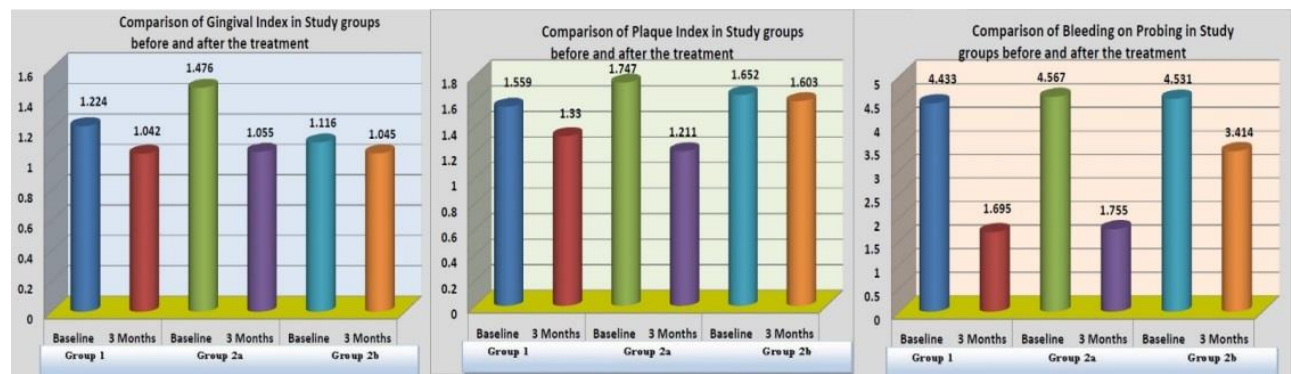

Fig. 3: Comparison of Gingival Index, Plaque Index and Bleeding on Probing in Study Groups before and after Treatment

\section{Discussion}

Psychosocial stress is being considered to play an important role in the pathogenesis of periodontal diseases and outcome of its treatment (Genco et al., 2008). Various mechanisms have been put forward to explain the role of stress in the development of periodontal disease. First, stress-induces a response that is transmitted to the hypothalamo-pituitary-adrenal (HPA) axis and promotes the release of corticotrophin-releasing hormone from the pituitary gland and glucocorticoid hormones from the adrenal cortex, which in turn induces the reduction of proinflammatory cytokines secretions (immunosuppressive effect). Second, exposure to stressor agents can induce the sympathetic nervous system to release adrenaline and noradrenaline which stimulates the formation and activity of prostaglandins and proteolytic enzymes, leading to periodontal tissue breakdown indirectly (Breivik and Thrane, 2001; Genco et al., 2008). Third, stress can induce the release of neuropeptides from sensory nerve fibers (neurogenic inflammation), whose presence has been implicated as a neurogenic promoter in various inflammatory processes modulating the activity of the immune system and the release of cytokines. Fourth, the behavioral model suggests that psychosocial stress may influence behavioral changes which affect health behaviors (i.e., negligent oral hygiene, intensification of smoking, poor compliance). Fifth, stress leads to overeating, especially high fat diets which increases cortisol production (Ross et al., 2014; Genco et al., 2008).

Contextually, studies to date imperatively suggest that if patients adapt successful coping strategies to reduce, control or tolerate the state of stress, there can be moderation on the impact of stress in the development of periodontal disease. Stressed periodontitis patients with maladaptive coping strategies (emotion focused coping-'avoidance') are at greater risk for developing severe periodontal disease and showed poorer response to a non-surgical periodontal treatment. Whereas, those with active coping strategies (problem solving coping'make a plan of action), are associated with high levels of wellbeing and found to have less periodontal tissues destruction and showed 
good response towards periodontal treatment. This suggests that a subject's responses to a stressor may be a key determinant in moderating the effect of stress on the progression of periodontal disease and outcome of the periodontal therapy (Wimmer et al., 2005).

If indeed stress and inadequate coping are important risk indicator for periodontal disease then to what extent intervention in stress reduction will have an effect in moderating these are unexplored till date. In view of this, the present study, which is first of its kind was undertaken to explore and evaluate intervention focused on employing psychological intervention (stress reduction therapy) as an adjunctive to the non-surgical periodontal therapy (NSPT). The patients were categorized as stressed or unstressed based on the salivary cortisol levels and DSP scores, as these are considered effective biological and psychological markers of stress (Vining et al., 1983). Studies have reported that the sources of stress and type of coping engaged by different genders and among the younger and elder individuals are categorically different; this might have an influence in the outcome of the study. Hence, to eliminate these confounding factors like age and gender differences, only the male, non-smoker individuals in the range of 30-55 years were recruited.

Since, the study participants were screened from Border Security Force (constitutes solely male population). the study group constituted only male participants.

In our study, transactional model of coping (Moss, 1999) was adapted, as a means of stress intervention. According to the Transactional Model, emotional and functional effects of primary and secondary appraisals are mediated by actual coping strategies. Original formulations of the model conceptualized coping efforts along two dimensions: (1) problem management and (2) emotional regulation. The problem-focused coping/problem-management strategies are directed at changing the stressful situation with the use of mechanisms such as active coping, problem solving, and information seeking. By contrast, emotion-focused coping efforts are directed at changing the way one thinks or feels about a stressful situation, by incorporating strategies such as seeking social support and venting feelings, as well as avoidance, and denial. The model predicts that problem-focused coping strategies will be most adaptive for stressors that are changeable, whereas emotion-focused strategies are most adaptive when the stressor is unchangeable or when this strategy is used in conjunction with problemfocused coping strategies. Irrespective of the coping style the patients were following, they were guided through the transactional model towards problem or emotional focused coping, as the situation warranted. Further, the patients were assessed periodically for their compliance and were directed towards effective stress management and oral hygiene behavior throughout the study period.

This indicates that the stressed patients undergoing NSPT + SRT demonstrated significantly higher associated reduction in the stress and clinical parameters when compared to stressed patients undergoing NSPT alone. It can also be observed that although the stress parameter reductions in Group 2a patients remained above the normal range, these patients with concomitant SRT showed a similar reduction in periodontitis affected sites as the unstressed patients. However, it was the unstressed Group 1 patients, who showed highest improvement in clinical parameters.

The mechanism whereby SRT may improve the outcome of periodontal treatment is not clear. The protocol of the present study included attempts to remodel patient's behavior and coping strategies along with non-surgical periodontal therapy, thus creating a three-sided approach towards disease management. Firstly, a more active participation in problem solving could have encouraged a more dynamic participation of the patients in day to day activities, including oral hygiene maintenance. Secondly, the decrease in oral inflammatory load after NSPT and periodic supportive periodontal therapy promoted decrease in periodontal inflammation. Thirdly, with the commencement of stress intervening programs, physiologic parameters favoring periodontal disease progression were controlled.

The results of our study are in similar lines with the previous studies, which propose that if the patient adapts to effective coping strategies, there could be moderation of the impact of stress on the periodontal tissues, thereby beneficially influencing the treatment outcomes as well. Wimmer et al. (2005) showed that passive coping strategies were more pronounced in advanced disease as well as in cases of poor response to a non-surgical periodontal treatment, whereas patients with active coping modes had milder disease and a more favorable course of treatment. Further, Gamboa et al. (2005) demonstrated that patients with good emotional intelligence (EI, a psychological construct) i.e., effective coping mechanism, is associated with favorable short-term changes in plaque and bleeding and the possibility of developing an intervention aimed at improving patients' EI was advised, which may improve response to initial treatment of periodontal disease. This suggests that subject's response to the stressors may be a key determinant of the overall effect of the stress.

Cortisol is a well-known stress-related hormone that can be detected in blood, saliva and gingival crevicular fluid (GCF). Salivary level of cortisol reliably reflects HPA axis activity and is used in human psychological studies as a biological marker of stress (Koh et al., 2007). In our study, salivary cortisol level was used as stress parameter because, it's an easy, non-invasive, rapid, stable, non-clotting medium, which requires less manpower and materials than other procedures. However, to compensate for diurnal variation, single-sample determination was done for all the patients at the same time of the day (Gharavi, 2008). The levels of cortisol in saliva were estimated by CLIA (glow-based chemiluminescent reaction) which provides a sensitive, high throughput, and economical alternative to conventional colorimetric methodologies, such as Enzyme-linked immunosorbent assays (ELISA). Further, it provides a broader dynamic assay range, superior low-end sensitivity, and a faster protocol than the conventional colorimetric methods (Gharavi, 2008). The DSP questionnaire is used to assess the degree of psychological stress, as it is one of the most comprehensive stress questionnaires designed to assess the chronic stress. The reliability of the questionnaire has been confirmed and it has also been proven that it requires no further random samples for standardization (Derogatis, 1995).

The three months follow up period adopted in the study was sufficient to demonstrate short-term changes in the clinical parameters. However, it is not sufficient to conclude for the long term and permanent changes. The results of the present study should thus, be interpreted with caution. The small, homogenous samples with short follow up duration, exclusion of female individuals, lack of random assignment to study groups, restriction to middle class or lower middle class families (those who were subjected to high strain and stress), limits the generalizability of the findings. Ideally, a study would use a set of biomarkers (Cortisol, DHEA, adrenaline, noradrenaline, dopamine and aldosterone) to measure stress response, while using questionnaires to measure stressor exposure and stress appraisal. In our study we used only a single stress biomarker (Cortisol) to objectively assess stress which could have led to some unknown confounding factors.

Future periodontal studies should be conducted with a larger sample size and longer follow up, including both male and female patients, with different lifestyles and family status, testing alternative psychological models of stress reduction in relation to adherence to treatment. Control groups should include lack of periodontal maintenance with various periodontal diseases. Integrated clinical, sociological and molecular based (biological markers, C-reactive protein) studies are needed very much for understanding the role of stress reduction therapy on the outcome of periodontal treatment.

\section{Conclusion}

To conclude, the result of our study is the first evidence to suggest that psychological intervention focused on stress reduction resulted in the same order of magnitude in the outcome as NSPT for the stressed chronic periodontitis patients when compared to unstressed chronic 
periodontitis patients. Hence, the finding of our study raises the possibility of developing an adjunctive intervention based on SRT for the holistic management of stressed patients undergoing periodontal treatment. However, more intervention studies are needed before psychological stress can be firmly established as been importance in the treatment or prevention of periodontal disease.

\section{References}

[1] Ainamo J and Bay I. Problems and proposals for recording gingivitis and plaque. International Dental Journal 1975; 25:229-235.

[2] Armitage GC. Development of a classification system for periodontal diseases and conditions. Annals of Periodontology 1999; 4:1-6. https://doi.org/10.1902/annals.1999.4.1.1.

[3] Bartold PM, Kylstra A and Lawson R. Substance P: An immunohistochemical and biochemical study in human gingival tissues. A role for neurogenic inflammation? Journal of Periodontology 1994; 65:1113-1121. https://doi.org/10.1902/jop.1994.65.12.1113.

[4] Breivik T, Thrane PS, Murison R and Gjermo P. Emotional stress effects on immunity, gingivitis and periodontitis. European Journal of Oral Sciences 1996; 104:327-334. https://doi.org/10.1111/j.1600-0722.1996.tb00087.x.

[5] Breivik T and Thrane PS. Psychoneuroimmune interactions in periodontal disease. Psychoneuroimmunology 2001; 2:627-644.

[6] Dawes C, Tsang RWL and Suelzle T. The effects of gum chewing, four oral hygiene procedures, and two saliva collection techniques, on the output of bacteria into human whole saliva. Archives of Oral Biology 2001; 46:625-632. https://doi.org/10.1016/S0003-9969(01)00017-6.

[7] Derogatis LR. The Derogatis stress profile (DSP): A summary report. http://www.derogatis-tests.com/dsp_synopsis.asp

[8] Dickerson SS and Kemeny ME. Acute stressors and cortisol responses: a theoretical integration and synthesis of laboratory research. Psychological Bulletin 2004; 130:355. https://doi.org/10.1037/0033-2909.130.3.355.

[9] Elter JR, Beck JD, Slade GD and Offenbacher S. Etiologic models for incident periodontal attachment loss in older adults. Journal of Clinical Periodontology 1999; 26:113-123. https://doi.org/10.1034/j.1600-051X.1999.260209.x.

[10] Genco RJ. Current View of Risk Factors for Periodontal Diseases. Journal of Periodontology 1996; 67:1041-1049. https://doi.org/10.1902/jop.1996.67.10.1041.

[11] Genco RJ, Ho AW, Kopman J, Grossi SG, Dunford RG and Tedesco LA. Models to evaluate the role of stress in periodontal disease. Annals of Periodontology 1998; 3:288-302. https://doi.org/10.1902/annals.1998.3.1.288.

[12] Gharavi MJ, Oormazdi H and Roointan ES. A comparative study on sensitivity and specificity of conventional and unconventional IgG and IgM assays for diagnosis of Toxoplasmosis. Iranian Journal of Public Health 2008; 37:42-45.

[13] Kiecolt-Glaser JK, Preacher KJ, MacCallum RC, Atkinson C, Malarkey WB and Glaser R. Chronic stress and age-related increases in the proinflammatory cytokine IL-6. Proceedings of the National Academy of Sciences 2003; 100:9090-9095. https://doi.org/10.1073/pnas.1531903100.

[14] Koh DSQ and Koh GCH. The use of salivary biomarkers in occupational and environmental medicine. Occupational and Environmental Medicine 2007; 64:202-210. https://doi.org/10.1136/oem.2006.026567.

[15] LeResche L and Dworkin SF. The role of stress in inflammatory disease, including periodontal disease: review of concepts and current findings. Periodontology 2000 2002; 30:91-103. https://doi.org/10.1034/j.1600-0757.2002.03009.x.

[16] Loe H and Silness J. Periodontal disease in pregnancy I. Prevalence and severity. Acta Odontologica Scandinavica 1963; $21: 533-551$. https://doi.org/10.3109/00016356309011240.

[17] Misra A, Chowbey P, Makkar BM, Vikram NK, Wasir JS, Chadha D and Munjal YP. Consensus statement for diagnosis of obesity, abdominal obesity and the metabolic syndrome for Asian Indians and recommendations for physical activity, medical and surgical management. Journal of the Association of Physicians of India 2009; 57:163-70.

[18] Moss D. Biofeedback, mind-body medicine, and the higher limits of human nature. Humanistic and transpersonal psychology: A historical and biographical sourcebook $1999 ; 145-161$.

[19] Page RC and Beck JD. Risk assessment for periodontal diseases. International Dental Journal 1997; 47:61-87. https://doi.org/10.1111/j.1875595X.1997.tb00680.x.

[20] Page RC. The pathobiology of periodontal diseases may affect systemic diseases: inversion of a paradigm. Annals of periodontology 1998; 3:108120. https://doi.org/10.1902/annals.1998.3.1.108.

[21] Pilgram TK, Hildebolt CF, Yokoyama-Crothers N, Dotson M, Cohen SC, Hauser JF and Kardaris E. Relationships between radiographic alveolar bone height and probing attachment level: data from healthy post-menopausal women. Journal of Clinical Periodontology 2000; 27:341-346. https://doi.org/10.1034/j.1600-051x.2000.027005341.x.

[22] Reners M and Brecx M. Stress and periodontal disease. International Journal of Dental Hygiene 2007; 5:199-204. https://doi.org/10.1111/j.16015037.2007.00267.x.

[23] Rosa EF, Corraini P, Inoue G, Gomes EF, Guglielmetti MR, Sanda SR, Lotufo JPB, Romito

[24] GA and Pannuti CM. Effect of smoking cessation on non-surgical periodontal therapy: Results after 24 months. Journal of Clinical Periodontology 2014; 41:1145-53. https://doi.org/10.1111/jcpe.12313.

[25] Seiffert K, Hosoi J, Torii H, Ozawa H, Ding W, Campton K and Granstein RD. Catecholamines inhibit the antigen-presenting capability of epidermal Langerhans cells. The Journal of Immunology 2002; 168:6128-6135. https://doi.org/10.4049/jimmunol.168.12.6128.

[26] Silness J and Loe H. Periodontal disease in pregnancy II. Correlation between oral hygiene and periodontal condition. Acta Odontologica Scandinavica $1964 ; 22: 121-135$. https://doi.org/10.3109/00016356408993968.

[27] Vining RF, McGinley RA, Maksvytis JJ and Ho KY. Salivary cortisol: a better measure of adrenal cortical function than serum cortisol. Annals of Clinical Biochemistry: An International Journal of Biochemistry in Medicine 1983; 20:329-335. https://doi.org/10.1177/000456328302000601.

[28] Wimmer G, Janda M, Wieselmann-Penkner K, Jakse N, Polansky R and Pertl C. Coping with stress: its influence on periodontal disease. Journal of Periodontology 2002; 73:1343-1351. https://doi.org/10.1902/jop.2002.73.11.1343.

[29] Wimmer G, Köhldorfer G, Mischak I, Lorenzoni M and Kallus KW. Coping with stress: Its influence on periodontal therapy. Journal of Periodontology 2005; 76:90-98. https://doi.org/10.1902/jop.2005.76.1.90. 